\title{
ADESÃO AO EXAME COLPOCITOPATOLÓGICO POR FUNCIONÁRIAS DO SERVIÇO PÚBLICO FEDERAL*
}

Dayse The Pereira ${ }^{1}$, Gleidson Brandão Oselame², Denecir de Almeida Dutra ${ }^{3}$, Elia Machado de Oliveira ${ }^{4}$, Eduardo Borba Neves $^{5}$

${ }^{1}$ Enfermeira. Centro Universitário Campos de Andrade. Curitiba, PR, Brasil.

${ }^{2}$ Enfermeiro. Mestre em Engenharia Biomédica. Docente de Enfermagem do Centro Universitário Campos de Andrade. Curitiba, PR, Brasil.

${ }^{3}$ Geógrafo. Doutor em Geografia da Saúde. Docente de Enfermagem do Centro Universitário Campos de Andrade Curitiba, $\mathrm{PR}$, Brasil.

${ }^{4}$ Enfermeira. Mestre em Cirurgia. Docente de Enfermagem do Centro Universitário Campos de Andrade Curitiba, PR, Brasil. ${ }^{5}$ Fisioterapeuta. Doutor em Saúde Pública. Docente pelo Programa de Pós Graduação em Engenharia Biomédica da Universidade Tecnológica Federal do Paraná. Docente pelo Programa de Pós Graduação em Engenharia Biomédica da Universidade Tecnológica Federal do Paraná. Docente pelo Centro Universitário Campos de Andrade. Curitiba, PR, Brasil.

RESUMO: Objetivou-se analisar o perfil de servidoras, em quatro órgãos do serviço público federal na cidade de Curitiba, estado do Paraná e suas percepções quanto à realização do exame colpocitopatológico. Tratouse de estudo descritivo, no ano de 2014, com 110 servidoras. Os resultados mostraram que 38,19\% ( $n=42)$ apresentavam idade superior a 50 anos. Entre os fatores de risco para o câncer de colo de útero, 7,27\% $(n=08)$ eram fumantes; $32,72 \%(n=36)$ não praticavam exercício físico; $26,36 \%(n=29)$ faziam uso de contraceptivos e $6,36 \%(n=07)$ não realizavam o exame preventivo conforme orientação médica. Sobre o exame $54,57 \%(n=60)$ responderam não apresentar nenhuma dificuldade. Relativo à visão das servidoras a respeito da patologia $38,19 \%$ $(\mathrm{n}=42)$ acreditavam ser importante para o diagnóstico precoce. Portanto, prevaleceram mulheres com idade superior aos 50 anos, não fumantes, praticantes de atividade física, sem uso de contraceptivos e que realizam o exame preventivo anualmente.

DESCRITORES: Neoplasias do colo do útero; Teste de papanicolaou; Educação em saúde.

\section{ADHERENCE TO CERVICAL SCREENING TEST BY SERVANTS OF THE FEDERAL PUBLIC ADMINISTRATION}

ABSTRACT: The present study aimed to analyze the profile of public servants from four bodies/agencies of the federal public administration, in the city of Curitiba, state of Paraná, and their perceptions of the cervical screening test. Descriptive study conducted in 2014 with 110 servants. The results showed that $38.19 \%(n=42)$ were older than 50 years. Regarding the risk factors for cervical cancer, $7.27 \%(n=08)$ were smokers; $32.72 \%(n=36)$ did not practice physical exercises; $26.36 \%(n=29)$ used contraceptives and $6.36 \%(n=07)$ did not get the routine cervical screening tests determined by the doctors. Regarding the test, $54.57 \%$ $(n=60)$ of the participants said they had no difficulty having cervical screening tests. Regarding the views of the servants on this pathology, $38.19 \%(n=42)$ believed it was important to get pap smear test to insure early detection of problems. Therefore, most servants were older than 50, non-smokers, practiced physical exercises, did not take contraceptives and had screening tests annually.

DESCRIPTORS: Cervical malignancies; Pap smear test; Health education.

\section{ADHESIÓN A UNA PRUEBA DE PAPANICOLAOU POR FUNCIONARIAS DEL SERVICIO PÚBLICO FEDERAL}

RESUMO: El objetibo del estudio fue analizar el perfil de funcionarias, en cuatro agencias del servicio público federal en la ciudad de Curitiba, estado de Paraná y sus percepciones cuanto a la realización del examen Papanicolaou. Es un estudio descriptivo, hecho en el año de 2014, con 110 funcionarias. Los resultados muestraron que $38,19 \% \quad(n=42)$ presentaban edad superior a 50 años. Entre los factores de riesgo para el cáncer de cuello de útero, 7,27\% ( $n=08)$ eran fumantes; $32,72 \% \quad(n=36)$ no practicaban actividad física; $26,36 \%(n=29)$ hacían uso de anticonceptivos y 6,36\% ( $n=07)$ no realizaban el examen de acuerdo a orientación médica. Sobre el examen $54,57 \%$ ( $n=60$ ) contestaron que no presentan ninguna dificultad. Acerca de la visión de las funcionarias sobre la patología 38,19\% ( $n=42)$ creen ser importante para el diagnóstico precoz. Por lo tanto, prevalecieron mujeres con edad superior a los 50 años, no fumantes, practicantes de actividad física, sin uso de anticonceptivos y que realizan el examen anualmente.

DESCRITORES: Neoplasias del cuello del útero; Prueba de Papanicolaou; Educación en salud.

*Artigo extraído de Trabalho de Conclusão de Curso de Graduação em Enfermagem, intitulado: “Adesão ao exame colpocitopatológico por funcionárias do serviço público federal. " Centro Universitário Campos de Andrade, 2014.

\section{Autor Correspondente:}

Gleidson Brandão Oselame

Centro Universitário Campos de Andrade

R. Mariano Torres, 958, ap 905 - 8000-120 - Curitiba, PR, Brasil

E-mail: gleidsonoselame@gmail.com
Recebido: $17 / 04 / 2015$ Finalizado: 25/06/2015 


\section{INTRODUÇÃO}

O rastreamento sistemático da população feminina por meio do exame colpocitopatológico do colo do útero, também conhecido como exame de Papanicolaou, tem sido uma das estratégias públicas mais efetivas, seguras e de baixo custo para detecção precoce desse câncer. O termo "Papanicolaou" é uma referência ao patologista grego Georges Papanicolaou, criador deste método no início do século. Atualmente, o exame Papanicolaou é a principal estratégia na detecção de lesões de colo uterino precocemente ${ }^{(1)}$.

O Ministério da Saúde do Brasil recomenda que toda mulher com vida sexual deverá submeterse ao exame preventivo periodicamente, especialmente aquelas com idade entre 25 e 59 anos. A indicação de realização é anual e após dois exames seguidos apresentando resultado normal, o preventivo pode ser feito a cada três $\operatorname{anos}^{(1)}$.

Dados do Instituto Nacional de Câncer (INCA) apontam o câncer de colo de útero como o terceiro tumor mais frequente na população feminina, atrás do câncer de mama e do colorretal, e a quarta causa de morte de mulheres por câncer no Brasil. A estimativa do INCA no biênio de 2014/2015 é de 15.590 novos $\operatorname{casos}^{(2)}$. Em relação aos países em desenvolvimento, como o Brasil, as taxas de incidência estimada e de mortalidade apresentam valores intermediários, já em países desenvolvidos estes valores são comparativamente elevados considerando seus programas de detecção precoce bem estruturados ${ }^{(3)}$.

Neste sentido, a Promoção a Saúde é uma ação essencial, pois é tida como o conjunto de ações dirigidas à saúde, por meio da ampliação do conhecimento da relação saúde-doença e trabalho. Especificamente, os servidores federais se beneficiam do Subsistema de Atenção à Saúde do Servidor (SIASS), o qual enfatiza o autocuidado durante os eventos promovidos internamente nos órgãos do serviço público federal que contribuam para a proteção da saúde no âmbito individual e coletivo. Desta forma, o presente estudo teve como objetivo analisar o perfil de servidoras do serviço público federal na cidade de Curitiba, estado do Paraná e suas percepções quanto à realização do exame colpocitopatológico.

\section{METODOLOGIA}

Tratou-se de um estudo descritivo transversal com análise qualiquantitativa dos dados. $\mathrm{O}$ estudo foi realizado na Agencia da Previdência Social Hauer (APS HAUER), no Instituto Nacional de Seguro Social (INSS), na Policia Rodoviária Federal $7^{a}$ Superintendência, e no Ministério da Agricultura, Pecuária e Abastecimento (MAPA), todos situados na cidade de Curitiba, estado do Paraná no ano de 2014. A amostra foi composta de forma aleatória por 110 servidoras do serviço público federal.

O instrumento utilizado para a coleta dos dados foi um questionário com nove questões fechadas e quatro abertas, que buscou identificar aspectos sociodemográficos, a visão da servidora frente ao câncer de colo do útero e os benefícios das ações que o Subsistema de Atenção à Saúde do Servidor (SIASS) promove internamente nos órgãos federais, por meio dos circuitos de saúde.

Para compor a amostra foram pré-estabelecidos os critérios de inclusão: ser servidora pública federal, maior de 20 anos e participar dos eventos promovidos pelo SIASS. Como critério de exclusão adotou-se servidoras que prestavam serviços terceirizados nos órgãos federais.

O questionário foi aplicado durante os eventos promovidos pelo SIASS, no período da manhã e tarde, durante o ano de 2014. Após serem respondidas as questões, as mulheres receberam um material explicativo sobre a importância do exame colpocitopatológico e os altos índices de cura quando o câncer de colo do útero é detectado precocemente. A análise quantitativa foi realizada por meio de tabelas de distribuição de frequência e a qualitativa pela metodologia da análise de conteúdo, na qual o pesquisador procura interpretar e identificar as categorias de análise que emergem das respostas dos participantes da pesquisa, para compreender o fenômeno que está sendo estudado ${ }^{(4)}$.

A pesquisa foi autorizada pela representante legal do SIASS. Foi garantido o sigilo das servidoras e demais conduta éticas em pesquisa envolvendo Seres Humanos conforme preconiza a resolução 466/2012 do Conselho Nacional de Saúde ${ }^{(5)}$. A pesquisa foi aprovada pelo Comitê de Ética em Pesquisa do Centro Universitário Campos de Andrade sob o parecer consubstanciado número 000581 de 2014.

\section{RESULTADOS}

Os dados foram categorizados em: Caracterização das mulheres; Fatores de risco para o desenvolvimento do câncer de colo uterino; A 
servidora federal frente à realização do exame preventivo; A problemática em realizar o exame colpocitopatológico; $\mathrm{O}$ saber das servidoras a respeito do câncer de colo uterino; e Eventos promovidos pelo SIASS visando à promoção em saúde dos servidores do serviço público federal.

\section{Caracterização das mulheres}

Responderam ao questionário um total de 110 mulheres. Destas, observou-se faixa etária predominante acima de 50 anos de idade $(n=42$; 38,19\%). A Tabela 1 apresenta as demais variáveis.

\section{Fatores de risco para o desenvolvimento do câncer de colo uterino}

Dentre os fatores de risco abordados prevaleceram as não fumantes $(n=102$; $92,73 \%$ ). Em relação à realização do exame colpocitopatológico $(n=103 ; 93,64 \%)$ realizam anualmente ou conforme orientação médica. As demais variáveis são apresentadas na Tabela 2 .

\section{A servidora federal frente à realização do exame preventivo}

Sobre a realização do exame colpocitopatológico e sua importância para a prevenção do câncer de colo de útero 38,19\% (n= 42) relatam ser importante na vida da mulher. As demais respostas são apresentadas na Tabela 3.

Os depoimentos abaixo descrevem a importância referida por $38,19 \%$ das mulheres em relação ao exame preventivo do câncer de colo do útero:

De suma importância para a mulher. (M31)

Importantíssimo, pois o câncer só tem cura quando se descobre no começo. (M14)

De extrema importância na prevenção da doença. (M 36)

Muito importante para um acompanhamento a respeito de como anda a saúde da mulher. (M 59)

É de máxima importância para prevenir a doença. (M74)

De fundamental importância. (M90)

Muito importante, faço sempre. (M86)

A realização do exame preventivo é muito importante, pois pode prevenir problemas futuros. (M 66)
Tabela 1 - Caracterização das servidoras do serviço público federal. Curitiba, PR, Brasil, 2014

\begin{tabular}{lcc} 
Variáveis & $\mathbf{n}$ & $\mathbf{\%}$ \\
\hline Faixa etária & & \\
\hline 20 a 30 anos & 11 & 10 \\
\hline 30 e 40 anos & 34 & 30,91 \\
\hline 40 e 50 anos & 23 & 20,90 \\
\hline$>50$ anos & 42 & 38,19 \\
\hline Tempo funcional federal & & \\
\hline$<5$ anos & 28 & 25,45 \\
\hline 05 a 10 anos & 17 & 15,45 \\
\hline 10 a 20 anos & 25 & 22,72 \\
\hline$>20$ anos & 40 & 36,38 \\
\hline Estado civil & & \\
\hline Solteira & 25 & 22,72 \\
\hline Casada & 56 & 50,93 \\
\hline Divorciada & 28 & 25,45 \\
\hline Viúva & 01 & 0,90 \\
\hline Número de filhos & & \\
\hline Nenhum & 35 & 31,83 \\
\hline Um filho & 27 & 24,54 \\
\hline Dois filhos & 32 & 29,09 \\
\hline$>03$ filhos & 16 & 14,54
\end{tabular}

Tabela 2 - Fatores de risco relacionado com o câncer de colo do útero. Curitiba, PR, Brasil, 2014

\begin{tabular}{lcc} 
Variáveis & $\mathbf{n}$ & $\mathbf{\%}$ \\
\hline Tabagista & 08 & \\
\hline Sim & 102 & 92,73 \\
\hline Não & & \\
\hline Faz uso de bebida alcoólica & 80 & 72,74 \\
\hline Socialmente & 0 & 0 \\
\hline Diariamente & 01 & 0,90 \\
\hline Mais de duas vezes na semana & 29 & 26,36 \\
\hline Não faz uso de bebida & & \\
\hline Pratica exercício físico & 16 & 14,54 \\
\hline Diariamente & 44 & 40,02 \\
\hline Duas a três vezes na semana & 14 & 12,72 \\
\hline Mais de quatro vezes & 36 & 32,72 \\
\hline Não pratica exercício & & \\
\hline Faz uso de anticoncepcional & 29 & 26,36 \\
\hline Sim & 81 & 73,64 \\
\hline Não & & \\
\hline $\begin{array}{l}\text { Realiza exame preventivo conforme } \\
\text { médica }\end{array}$ & 103 & 93,64 \\
\hline Sim & 07 & 6,36 \\
\hline Não & &
\end{tabular}


Tabela 3 - Opinião das servidoras do serviço público federal a respeito da realização do exame preventivo. Curitiba, PR, Brasil, 2014

\begin{tabular}{lcc} 
Variáveis & $\mathbf{n}$ & $\mathbf{0}$ \\
\hline Necessário & 29 & 26,36 \\
\hline Importante & 42 & 38,19 \\
\hline Forma de prevenção & 32 & 29,09 \\
\hline Não responderam & 07 & 6,36
\end{tabular}

\section{A problemática em realizar o exame colpocitopatológico}

Sobre as possíveis dificuldades enfrentadas na realização do exame colpocitopatológico, observou-se que $54,57 \% \quad(n=60)$ relataram não ter nenhuma dificuldade em realizar o exame. As demais variáveis são apresentadas na Tabela 4.

Tabela 4 - Dificuldade em realizar o exame preventivo. Curitiba, PR, Brasil, 2014

\begin{tabular}{lcc} 
Variáveis & $\mathrm{n}$ & $\%$ \\
\hline Desconforto/vergonha & 9 & 8,18 \\
\hline Não se lembra de fazer o exame & 3 & 2,72 \\
\hline Falta de tempo & 24 & 21,81 \\
\hline Nenhuma dificuldade & 60 & 54,57 \\
\hline Não responderam & 14 & 12,72
\end{tabular}

Os relatos a seguir ilustram que $54,57 \%$ das mulheres não referem dificuldades em relação ao exame preventivo:

Não tenho dificuldade, realizo através do plano de saúde. (M 33)

Não tenho. (M 45)

Não sinto dificuldade. (M106)

Não tenho dificuldades quanto à realização do exame. (M 108)

\section{O saber das servidoras a respeito do câncer de colo uterino}

Observou-se que $36,38 \%(n=40)$ relataram que se diagnosticado precocemente, a chance de cura aumenta significativamente. As demais respostas são representadas na Tabela 5 .

Observa-se que a maioria acredita ser importante o diagnóstico precoce como revelam os depoimentos a seguir:

Não conheço os detalhes, apenas que pode ser
Tabela 5 - A visão das servidoras a respeito do câncer de colo do útero. Curitiba, PR, Brasil, 2014

\begin{tabular}{lcc} 
Variáveis & $\mathrm{n}$ & $\%$ \\
\hline $\begin{array}{l}\text { Diagnóstico precoce e chance de } \\
\text { cura }\end{array}$ & 40 & 36,38 \\
\hline $\begin{array}{l}\text { HPV (Papiloma vírus humano) e } \\
\text { câncer }\end{array}$ & 7 & 6,36 \\
\hline Doença grave & 8 & 7,27 \\
\hline $\begin{array}{l}\text { Sabe pouco sobre o câncer do } \\
\text { colo do útero }\end{array}$ & 22 & 20 \\
\hline $\begin{array}{l}\text { Uma das principais causa de } \\
\text { morte em mulheres }\end{array}$ & 9 & 8,18 \\
\hline Não responderam & 24 & 21,81
\end{tabular}

identificado com o preventivo. (M48)

Somente sei que o preventivo é fundamental para detectá-lo em estágio curável. (M93)

Se faz necessário prevenção, realizar exame anualmente pra que haja chance de cura. (M05)

É um câncer bastante agressivo que se não for atendido em seu início pode levar até à morte. (M88)

Prevenção é o melhor... Cuidar-se. (M54)

Que é um mal que pode ser evitado desde que se faça prevenção. (M61)

Que o exame preventivo pode evitar ou mesmo proporcionar diagnóstico em fases iniciais, que atualmente também há alguns tipos que podem ser prevenidos com vacina. (M100)

Com a internet facilita o conhecimento, prevenir ainda é a melhor atitude. (M106)

\section{Eventos promovidos pelo SIASS visando à promoção da saúde dos servidores federais}

Os circuitos da saúde que o grupo SIASS realiza nos órgãos do serviço público federal têm como principal objetivo a conscientização das servidoras na promoção da saúde. Há várias equipes atuantes neste processo: enfermagem, nutrição, massoterapeutas, entre outros, desenvolvendo atividades educativas.

Neste sentido, observou-se que 40,93\% das servidoras $(n=45)$ relatam a importância destes eventos para a conscientização de hábitos saudáveis. Outras $(n=25 ; 22,72 \%)$ descrevem a ênfase na qualidade devida, enquanto que $22(20 \%)$ relatam ser excelente para a saúde ocupacional e $(\mathrm{n}=05 ; 4,54 \%)$ ser eficaz na prevenção de várias 
patologias devido às orientações recebidas pelos profissionais. Apenas $11,81 \% \quad(n=13)$ não responderam essa questão, pois estavam participando pela primeira vez do evento.

Constatou-se que a maior parte das entrevistadas alega que os eventos realizados nos órgãos federais, por meio do SIASS, desenvolvem a conscientização para hábitos saudáveis como representado nos relatos a seguir:

Acho de grande importância, pois acredito que a prevenção é sempre o melhor caminho. (M22)

Importante para a conscientização do cuidado preventivo que devemos ter conosco. (M48)

Muito importante, deveria ser mais frequente. (M31)

Importante, ter sempre palestra todo o ano. (M102)

\section{É importante para todos. (M88)}

Importante, pois no dia a dia não temos muito tempo para nos preocuparmos com a saúde. (M75)

Importante, pois acabamos por negligenciar nossa saúde. (M50)

De grande importância para nossos servidores, pois no dia a dia do trabalho não prestamos muito atenção à nossa saúde, trata-se de uma oportunidade para melhorar nossa qualidade de vida. (M110)

\section{DISCUSSÃO}

Constatou-se que a maioria das mulheres possui idade superior a 50 anos. Dados semelhante a um estudo realizado na cidade de Uberaba, Minas Gerais, com 335 mulheres, no qual as idades variaram de 54 a 90 anos, em que 91,6\% sabiam perfeitamente da importância à adesão ao exame e seus benefícios ${ }^{(6)}$.

Por se tratarem de servidoras federais, supõe-se que o nível de escolaridade pode ter contribuído para maior adesão ao exame preventivo. Isto é importante, pois este indicador pode potencializar os fatores de risco para o desenvolvimento de doenças, não somente pela precária condição de moradia e alimentação, mas também pela adesão das mulheres às medidas preventivas de saúde ${ }^{(7)}$.

Tal ocorrência é reforçada em um estudo realizado em Belo Horizonte, Minas Gerais, no qual foram incluídas 1214 mulheres, sendo a frequência de realização do exame de $76,43 \%$. Os resultados descaram que, entre outras variáveis, possuir baixa escolaridade demonstrou associação com a não realização do exame ${ }^{(8)}$. Outro estudo realizado no estado da Bahia, no qual foram entrevistadas 230 mulheres, observou que a não adesão ao exame foi significativamente superior entre aquelas que nunca frequentaram a escola ${ }^{(9)}$.

No que diz respeito ao tempo de funcionalismo público houve uma prevalência de mais de 20 anos exercendo a profissão. Isso demonstra que uma importante alteração tem ocorrido no campo de trabalho da mulher brasileira e que, na medida em que as mulheres aumentam sua presença no mercado de trabalho, ocorrem alterações em seus papéis, estilos de vida e padrões de cuidados com a saúde ${ }^{(10)}$.

A maioria das mulheres entrevistadas era casada e com um a três filhos. Isto pode ser considerado um dos fatores de risco associados ao câncer de colo de útero. No entanto, a condição de não ter filhos também pode ser associada a não realização do exame preventivo(7). Outro estudo destaca que a adesão ao exame preventivo foi maior nas mulheres com quatro ou mais filhos ou com histórico de quatro ou mais partos ${ }^{(9)}$.

Destaca-se ainda que o estado civil esteja associado à melhora da saúde das pessoas e das populações de um modo geral, pois confere certa proteção e segurança favorável à mulher, que se refletem diretamente no seu nível de saúde. A vida a dois costuma trazer mudanças positivas nos comportamentos de saúde e sexual do casal. Em geral, alteram-se os riscos venéreos, e a gravidez pode ser mais planejada, conferindo estabilidade, qualidade de vida e proteção ao casal para lidar com os problemas da vida moderna ${ }^{(10)}$.

Em um estudo realizado com 51 mulheres no estado do Piauí, $66,7 \%$ delas eram casadas e $55 \%$ acessaram os serviços de saúde buscando a prevenção do câncer do colo de útero pelo exame Papanicolaou ${ }^{(11)}$. Tal resultado difere de outro, no qual as mulheres compreendiam o exame preventivo de forma superficial, com enfoque maior no aspecto curativo em relação ao preventivo $^{(12)}$.

No presente estudo prevaleceram mulheres não tabagistas, com ingestão de bebidas alcoólicas socialmente, praticantes de exercícios físicos de duas a três vezes por semana e não fazendo uso de contraceptivos. Isto indica que a amostra pesquisada apresentava hábitos de vida importantes na prevenção do câncer do colo do útero, pois mulheres que ingerem bebida 
alcoólica mais de duas vezes e que fumam de 11 a 30 cigarros por dia apresentam elevados índices de alteração cervical. Ainda, o uso de contraceptivos por um longo período de tempo (5 a 9 anos) aumenta significativamente o risco para o câncer de colo de útero ${ }^{(13)}$.

Por outro lado, a atividade física mostra-se preventiva ao câncer por ativar mecanismos biológicos atuantes no sistema imunológico, por meio do aumento de enzimas atuantes nos radicais livres, as quais podem inibir a formação do tumor $^{(14)}$. Diferentemente da amostra pesquisada, um estudo encontrou em 51 mulheres, 41,2\% com sobrepeso e 19,6\% com obesidade. Dentre estas, $72,5 \%$ eram sedentárias ${ }^{(11)}$. Neste sentido, observa-se que as mulheres avaliadas no presente estudo desenvolvem atividades importantes na prevenção do câncer do colo do útero.

A maior parte das servidoras federais realizava e acreditava ser importante o exame preventivo, tal fato ficou evidente pelos discursos apresentados. Este achado difere do encontrado em outro estudo, em que foram avaliadas 200 mulheres no estado de Minas Gerais, das quais a maioria possuía déficit de conhecimento em relação ao objetivo do exame ${ }^{(15)}$. Ressalta-se que a realização periódica do exame é indispensável, visto que quando a doença é diagnosticada precocemente e tratada adequadamente, existe $100 \%$ de chance de cura ${ }^{(7)}$.

E ainda, o medo do resultado do exame colpocitopatológico e a vergonha de se expor ao profissional de saúde são os fatores que mais exercem influência na decisão das mulheres em realizar ou não o exame. Um estudo realizado no estado do Paraná, com 81 mulheres, destaca que para $65,61 \%$ delas os motivos para não realizarem o exame são por vergonha do profissional de saúde ou por medo do resultado do exame ${ }^{(7)}$.

Um estudo realizado com 35 servidoras federais no estado do Ceará destacou situações como medo e vergonha na realização do exame ${ }^{(16)}$. Porém, constatou-se no presente estudo que a maioria das mulheres não apresenta nenhuma dificuldade na realização do exame, o que é positivo e sugere bom entendimento sobre a relevância do método de prevenção.

Por fim, observou-se grande participação das mulheres em palestras que versam sobre a importância do exame em eventos do SIAAS que objetivam atender e padronizar a atenção à saúde por meio da promoção, prevenção e acompanhamento dos servidores da administração federal ${ }^{(17)}$. As ações realizadas enfatizam a importância na prevenção de agravos à saúde, sobretudo aqueles voltados ao campo de saúde da mulher.

\section{CONCLUSÃO}

Dentre as mulheres avaliadas no estudo, prevaleceram aquelas com idade superior aos 50 anos, não fumantes, praticantes em sua maioria de atividade física regular, sem uso de contraceptivos e que realizam o exame preventivo para o câncer do colo do útero anualmente.

Observou-se que a percepção destas mulheres em relação ao exame foi direcionada a redução dos riscos em relação ao câncer de colo de útero, destacando o bom entendimento sobre a importância de ações preventivas realizadas no SIASS e por grande parte não apresentar dificuldades em se submeter à realização do exame preventivo.

\section{REFERÊNCIAS}

1. Instituto Nacional do Câncer (INCA). Exame preventivo do câncer de colo uterino (Papanicolau) INCA [Internet] 2011 [acesso em 22 jun 2015]. Disponível: http://bvsms.saude.gov.br/bvs/dicas/237_ papanicolau.html

2. Ministério da Saúde (BR). Instituto Nacional de Câncer José Alencar Gomes da Silva (INCA) [Internet]. Estimativa 2014: incidência de câncer no Brasil-Regiões/ consolidado. Rio de Janeiro [acesso em 17 jun 2015]. Disponível: http://www.inca.gov.br/estimativa/2014/ tbregioes_consolidado.asp

3. Ministério da Saúde (BR). Instituto Nacional de Câncer José Alencar Gomes da Silva (INCA) [Internet]. Controle do câncer de colo de útero. Rio de Janeiro [acesso em 22 jun 2015]. Disponível: http://www2. inca.gov.br/wps/wcm/connect/acoes_programas/site/ home/nobrasil/programa_nacional_controle_cancer_ colo_utero/conceito_magnitude

4. Gil AC. Métodos e técnicas de pesquisa social. São Paulo: Atlas; 2010.

5. Ministério da Saúde (BR). Conselho Nacional de Saúde. Diretrizes e normas regulamentadoras de pesquisa envolvendo seres humanos. Resolução $n$. 466, [Internet] 12 de dezembro [acesso em $22 \mathrm{fev}$ 2014]. Disponível: http://conselho.saude.gov.br/ resolucoes/2012/Reso466.pdf

6. Maeda TC, Alves AP, da Silva SR. Conhecimento de mulheres idosas sobre o exame de papanicolaou. Ciênc. saúde coletiva. 2013; 11(2):360-7. 
7. Teilo MA, Oselame GB, Dutra DDA, Neves EB. Fatores relacionados à adesão do exame colpocitopatólogico no munícipio de cerro azul. Ciênc. saúde coletiva. 2014; 13(1):90-6.

8. Lage AC, Pessoa MC, Meléndez JGV. Fatores associados à não realização do Teste de Papanicolaou em Belo Horizonte, Minas Gerais, 2008. Reme, Rev Min Enferm. 2013; 17(3):565-76.

9. Andrade MS, de Almeida MMG, Araújo TM, Santos KOB. Fatores associados a não adesão ao Papanicolau entre mulheres atendidas pela Estratégia Saúde da Família em Feira de Santana, Bahia, 2010. Epidemiol serv saúde. 2014; 23(1):111-20.

10. da Silva MRB, da Silva LGP. O conhecimento, atitudes e prática na prevenção do câncer uterino de uma unidade da zona oeste-Rio de Janeiro. Rev Pesqui: Cuid Fundam. [Internet] 2012; 4(3) [acesso em $10 \mathrm{dez}$ 2014]. Disponível: http://arca.icict.fiocruz.br/handle/ icict/9169

11. Oliveira AC, Pessoa RS, de Carvalho AMC, Magalhães RLB. Fatores de risco e proteção à saúde de mulheres para prevenção do câncer uterino. Rev Rene. 2014; 15(2):240-8.

12. Oliveira $\mathrm{SL}, \mathrm{Almeida} \mathrm{ACH}$. A percepção das mulheres frente ao exame de Papanicolaou: da observação ao entendimento; La percepción de las mujeres frente al examen de papanicolaou: de la observación a la comprensión. Cogitare enferm. 2009; 14(3):518-26.

13. dos Anjos SJSB, Vasconcelos CTM, Franco ES, de Almeida PC, Pinheiro AKB. Fatores de risco para câncer de colo do útero segundo resultados de IVA, citologia e cervicografia. Rev Esc Enferm USP. 2010; 44(4):912-20.

14. Pedroso W, Araújo MB, Stevanato E. Atividade física na prevenção e na reabilitação do câncer. Motriz. 2005; 11(3):155-60.

15. Mendes LC, Silveira CF, da Silva SR. Conhecimento de mulheres a respeito do exame de papanicolaou e do autoexame das mamas. REAS. 2013; 2(3):4-17.

16. Jorge RJB, Diógenes MAR, da Cruz Mendonça FA, Sampaio LRL, Júnior RJ. Exame Papanicolaou: sentimentos relatados por profissionais de enfermagem ao se submeterem a esse exame. Ciênc saúde coletiva. 2011; 16(5):2443-51.

17. Schuster MS, Dias VV, Battistella LF. Unidade SIASS Santa Maria: Uma proposta empreendedora para sua Implantação. UNOPAR Cient, Ciênc Juríd Empres. 2014; 15(1):61-9. 